\title{
LA TONADILLA ESCÉNICA EN VENEZUELA O EL PROCESO DE CRIOLLIZACIÓN DE UN GÉNERO HISPANO
}

\author{
THE STAGE TONADILLA IN VENEZUELA OR THE PROCESS OF \\ CREOLIZATION OF A HISPANIC MUSICAL GENRE
}

\author{
Montserrat Capelán Fernández \\ Universidade de Santiago de Compostela \\ aulos90@gmail.com \\ ORCID iD: http://orcid.org/0000-0001-6007-1538
}

\begin{abstract}
Resumen
En el presente trabajo se estudia la interpretación de la tonadilla escénica tanto en la época colonial, en el que se representaban obras de origen peninsular, como en la republicana, cuando ya autores locales empiezan a dedicarse a este género. Se establecerán las características estructurales de lo que la prensa denominaba tonadilla y lo que llamaba opereta y, contraviniendo a José Subirá, se pondrá de manifiesto que la tonadilla no sufre un proceso de italianización sino, muy por el contrario, de hispanización. Una vez llegada la República y con ella el cultivo de la tonadilla escénica por autores venezolanos, este proceso dará lugar a una criollización introduciéndose en la tonadilla escénica bailes autóctonos como el merengue. En el presente texto haremos un estudio detallado de él, y estableceremos la posibilidad de que el origen del merengue, esté en las tiranas de las tonadillas peninsulares del siglo anterior.
\end{abstract}

\section{Palabras clave}

Tonadilla escénica, criollización, tirana, merengue, Venezuela, Blas de Laserna, José Ángel Montero, Atanacio Bello Montero.

En 1792 Manuel Torres y José María Castro, asentistas del Coliseo de Caracas, escriben una misiva en la que señalan tener muchos gastos, entre otras cosas, por la necesidad de representar y ensayar tonadillas en los intermedios de las comedias presentadas ${ }^{1}$. Se trata éste del primer documento

1 Así se expresaban Torres y Castro en una carta al Ayuntamiento: "el costo [de las representaciones] siempre es uno mismo, y no pequeño [...] que habiendo nosotros de procurar el desempeño a satisfacción de los espectadores, no sólo hemos de ofrecer una orquesta

\begin{abstract}
The present study examines the performance of the stage tonadilla during the colonial period, when works originating from the peninsula were performed, as well as during the Republic, when local composers began cultivating this genre. We shall establish the structural characteristics of what the press called tonadilla and what was considered operetta and, in disagreement with José Subirá, it will become evident that the tonadilla does not undergo a process of Italianization but rather of a marked Hispanic slant. Once the Republic is installed and along with it the cultivation of the stage tonadilla by Venezuelan composers, this process will give way to a creolization introducing within the stage tonadilla local dances such as the meringue. We shall undertake a detailed study of this, and we shall establish the possibility that the origin of the meringue is in the tiranas of the peninsular tonadillas of the previous century.
\end{abstract}

\section{Key words}

Stage tonadilla, creolization, tirana, meringue, Venezuela, Blas de Laserna, José Ángel Montero, Atanacio Bello Montero.

venezolano en el que se menciona el género. Si tomamos en cuenta, sin embargo, en que se habla de la tonadilla como algo ya asentado, hemos de asumir que éstas se empezaron a representar unos años atrás. La fecha más probable es 1789 , momento a partir del cual en la plantilla del Coliseo caraqueño

razonable y hemos de dar Tonadillas, sino también pagar sus ensayos y ejecución". 6 de febrero de 1792, Archivos Histórico del Concejo Municipal, Caracas (AHCM), Coliseo, tom. I, n ${ }^{\circ} 6$. 
empezamos a encontrar mujeres cantantes. Con ello podemos decir que la tonadilla escénica se popularizó en Venezuela, unos 18 años después de estar en pleno apogeo en Madrid².

A partir de esta época se volverá habitual la interpretación de tonadillas en los intermedios de las comedias que eran presentadas no sólo en el Coliseo caraqueño, sino también en los patios de comedias e, incluso, en las casas particulares en las que se hacían representaciones. Una muestra de lo primero, es la presentación en un patio de Guatire (población próxima a Caracas) de cinco comedias y dos tonadillas organizadas por el músico Juan Bautista Olivares ${ }^{3}$. Por su parte, en las casas particulares, se representaban aquellas tonadillas escénicas cuya temática contravenía a la corriente neoclásica, motivo por el cual eran prohibidas en el Coliseo de la ciudad ${ }^{4}$.

Hasta 1821, momento en que Venezuela consigue la independencia definitiva de la corona española, conocemos los títulos de 15 tonadillas interpretadas en Caracas. Excepto dos obras a las que no le hemos podido atribuir autor, todas son composiciones de los españoles Blas de Laserna o Pablo del Moral, con una clara predilección por el primero.

\begin{tabular}{|c|c|c|c|c|}
\hline AUTOR & TÍTULO & GÉNERO $^{5}$ & FUENTE & FECHA \\
\hline Blas de Laserna & La viuda de seis maridos & Tonadilla a solo* & $\begin{array}{c}\text { Gazeta de Caracas, } \\
3 / 12 / 1811,6 / 12 / 1811\end{array}$ & $8 / 12 / 1811$ \\
\hline Pablo del Moral & El contrato matrimonial & $\begin{array}{c}\text { Tonadilla a } 3 \\
\text { Opereta* }\end{array}$ & $\begin{array}{l}\text { Gazeta de Caracas, } \\
3 / 12 / 1811,6 / 12 / 1811\end{array}$ & $8 / 12 / 1811$ \\
\hline Blas de Laserna & Mas no quiero murmurar & Tonadilla a solo* & $\begin{array}{c}\text { Gazeta de Caracas, } \\
13 / 12 / 1811 \\
\text { AABN Caja } 8 \text { N } 138 \\
\end{array}$ & $15 / 12 / 1811$ \\
\hline--- & $\begin{array}{l}\text { A que una dama se burla de } \\
\text { dos hombres a un tiempo }\end{array}$ & Tonadilla a tres* & $\begin{array}{c}\text { Gazeta de Caracas } \\
13 / 12 / 1811\end{array}$ & $15 / 12 / 1812$ \\
\hline Blas de Laserna & El médico y sus (los) sobrinos & $\begin{array}{c}\text { Tonadilla a tres } \\
\text { Opereta* }\end{array}$ & $\begin{array}{c}\text { Gazeta de Caracas, } \\
10 / 1 / 1812 \\
\end{array}$ & $\begin{array}{c}6 / 1 / 1812 \\
12 / 1 / 1812 \\
\end{array}$ \\
\hline Pablo del Moral & El amor por poderes & $\begin{array}{c}\text { Tonadilla a tres } \\
\text { Opereta* }\end{array}$ & $\begin{array}{c}\text { Gazeta de Caracas } \\
14 / 1 / 1812 \\
\end{array}$ & $19 / 1 / 1812$ \\
\hline Blas de Laserna & Los frenos trocados & Tonadilla a solo & AABN CCU 0888 & --- \\
\hline Blas de Laserna & La civilización & Tonadilla a solo & AABN CCU 0882 & --- \\
\hline Blas de Laserna & La italiana y la andaluza & Tonadilla a 3 & $\begin{array}{c}\text { AABN Manuscritos sin } \\
\text { ingreso en la base de datos } \\
\text { Caja } 4 \text { N } 55\end{array}$ & --- \\
\hline Blas de Laserna & El enemigo de las mujeres & Tonadilla a dúo & AABN sin catalogar & --- \\
\hline Blas de Laserna & El asturiano burlado & Tonadilla a tres & AABN sin catalogar & --- \\
\hline--- & Músico y el poeta ${ }^{6}$ & Tonadilla a tres & $\begin{array}{l}\text { AABN Sin catalogar Caja } \\
21 \text { N } 356\end{array}$ & --- \\
\hline
\end{tabular}

4 Cuando menos desde 1801 en el Coliseo caraqueño fue prohibida la representación de comedias que contraviniesen el estilo neoclásico. Por ello cabe esperar que las tonadillas que iban en contra de esta corriente y que sabemos que se interpretaron en Caracas en la época colonial, se llevaran a cabo fuera de este recinto teatral, seguramente en las casas en las que sí se interpretaba el repertorio barroco que estaba vedado en el Coliseo (CAPELÁN, 2015: vol. 1, 325-339). En referencia a la interpretación de tonadillas en casas particulares en las ciudades peninsulares de Barcelona y Madrid puede consultarse PESSARRODONA, 3 (Valencia, 2014): 123 y SUBIRÁ (1928): vol. 1, 264-272.

5 Se indican con asterisco los nombres con los que aparecen in-

2 No es su introducción demasiado tardía, si tomamos en cuenta que su representación en Nueva España se vuelve habitual en 1786 y que, en Santiago de Chile, recibe su "aprobación oficial" en 1793 (VERA, 2016: 3; CLARO, 156 (Santiago de Chile, 1981): 15).

3 Ejecución que sigue Juan Bautista Olivares contra Joaquín Xedler por el cobro de 50 pesos, 1793-1794, Academia Nacional de la Historia, Caracas (ANH), Civiles, 10-3980-3, f. 1. dicadas en la Gazeta de Caracas.

6 No coincide musicalmente con la obra homónima (también conocida como Los Maestros de la Raboso) de Carnicer. Dado que sólo se conservan las particellas de oboe 2 y violín 1 es imposible saber si se utilizó el mismo libreto de la obra de Carnicer o no. 


\begin{tabular}{|c|l|c|c|c|}
\hline Blas de Laserna & El Amante rendido & Tonadilla a dúo & $\begin{array}{c}\text { AABN Manuscritos sin } \\
\text { ingreso en la base de datos } \\
\text { caja 19 N 323 }\end{array}$ & $\begin{array}{c}\text {--- } \\
\text { Pablo del Moral }\end{array}$ \\
\hline El hombre mujer & Tonadilla a dúo & $\begin{array}{c}\text { AABN Manuscritos sin } \\
\text { ingreso en la base de datos } \\
\text { caja 4 N 54 }\end{array}$ & $\begin{array}{c}\text { AABN Manuscritos sin } \\
\text { ingreso en la base de } \\
\text { datos. Caja compositores } \\
\text { venezolanos por catalogar e } \\
\text { identificar }\end{array}$ \\
\hline
\end{tabular}

Tabla 1. Tonadillas escénicas que fueron representadas en la Venezuela colonial de las que conocemos los títulos.

En las partituras actualmente conservadas podemos observar, gracias a la caligrafía, que el material era importado directamente de la Península. Llama la atención que, en ocasiones, incluso se indiquen los nombres de las intérpretes madrileñas, como ocurre con La italiana y la andaluza de Blas de Laserna, en cuya partitura de la Biblioteca Nacional de Venezuela se nombra a las cantantes Antonia Prado y Mariana Márquez, cantantes de los coliseos madrileños ${ }^{7}$.

En todo caso, lo realmente importante son las diferencias (algunas mínimas pero otras de importancia considerable) entre las versiones conservadas en Venezuela y las que están en Madrid. Las madrileñas son, en muchos casos, versiones preliminares que se utilizaban en las prime- ras representaciones de cada tonadilla mientras que, las que actualmente están en Caracas, son versiones finales que se pasaban a limpio cuando ya las tonadillas habían alcanzado cierto rodaje.

Las discrepancias más interesantes las encontramos cuando se presentan en una versión, números completamente nuevos. Por ejemplo, en La italiana y la andaluza conservada en Caracas de Blas de Laserna, se incluyen unas seguidillas distintas a las que se encuentran en la Biblioteca Histórica de Madrid. Más llamativo todavía es el cambio de El asturiano burlado, del mismo autor, en donde en Caracas tenemos un segundo violín completamente diferente y tres movimientos ajenos a los de la versión española.

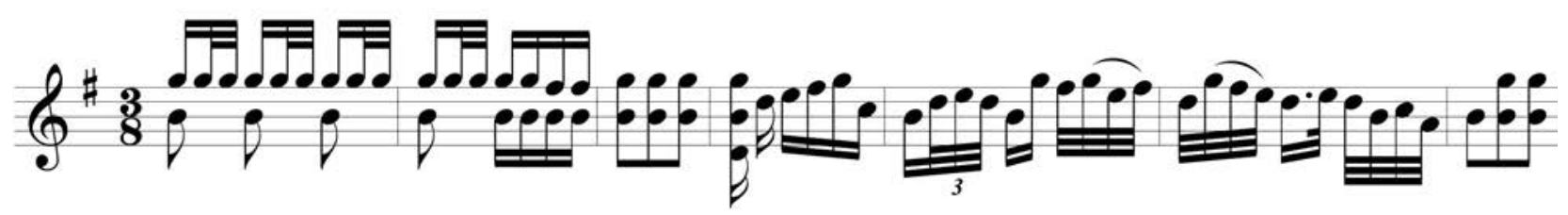

Figura 1. Violín $1^{\circ}$ en el quinto movimiento de La italiana y la andaluza de Laserna conservado en Madrid (boleras) (BHM, Mus 129-12)

7 Archivo Audiovisual de la Biblioteca Nacional de Venezuela, Caracas (AABN), Manuscritos sin ingreso en la base de datos, Caja $4, \mathrm{~N}^{\circ} 55$. Este dato no deja de ser importante, pues en los manuscritos conservados en España no se indica cuáles fueron las cantantes de esta tonadilla, motivo por el cual José Subirá asegura desconocer quiénes cantaron esta tonadilla en Madrid (SUBIRÁ, 1929: vol. 2, 139). 


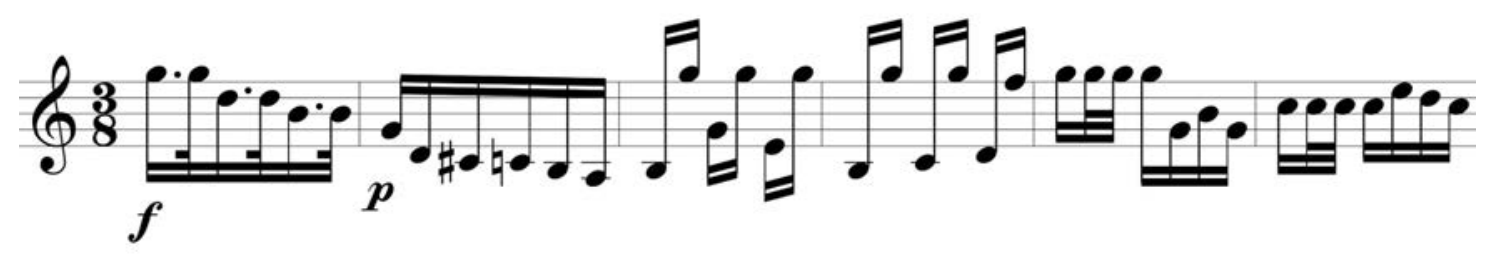

Figura 2. Violín $1^{\circ}$ en el quinto movimiento de La italiana y la andaluza de Laserna (seguidillas boleras) conservado en Caracas en el que se puede apreciar la diferencia con el madrileño (AABN, Manuscritos sin catalogar, caja $4 \mathrm{~N}^{\circ}$ 55).

\section{LA TONADILLA ESCÉNICA DURANTE LA ÉPOCA COLONIAL: DE LA TONADILLA A LA OPERETA}

El 6 de diciembre de 1811, después de un período de clausura del Coliseo caraqueño debido a la situación bélica, encontramos el siguiente anuncio en la prensa caraqueña:

Habiendo S. A. el Supremo poder ejecutivo dispuesto, que pueda abrirse el coliseo y principarse las funciones se convida al público para el Domingo ocho del presente, día de la Concepción, si el tiempo lo permite a una famosa Comedia en Tres Actos, titulada / Morir por la patria es gloria; / acabado el primer acto se cantará la primorosa tonadilla a solo / La Viuda de seis Maridos; / después del segundo se cantará, / La Opereta, / o el / Contrato Matrimonial, / luego una buena / Canción patriótica Nueva, / y si no fuere tarde de dará un gracioso / Sainete ${ }^{8}$.

El anuncio no tendría nada de particular a no ser porque en él se establece una diferencia de género entre $L a$ viuda de seis Maridos (tonadilla) y El contrato matrimonial (opereta). Con ello encontramos en la prensa una distinción inexistente en las partituras, en las que se denomina a las dos tonadillas por igual.

En esta sección nos preguntaremos si existía una diferencia estructural entre lo que la prensa denominaba tonadillas y lo que llamaba operetas. Indicaremos cómo se desarrolló el cambio de un género a otro así como demostraremos que la tonadilla escénica no sufrió un proceso de italianización como José Subirá aseguraba, sino que, muy por el contrario, se dio un proceso de hispanización con el que se pretendía competir con el auge de la ópera italiana.

A este respecto es importante recordar el memorial que el compositor Blas de Laserna dirigirá a la Junta de formación en 1792, quejándose del excesivo trabajo que se le impone y en el que manifiesta que las antiguas tonadillas han

8 Gazeta de Caracas, 6 de diciembre de 1811, p. 4. sido poco a poco sustituidas por obras mucho más largas que son verdaderas operetas:

[Las tonadillas estaban] compuestas de introducción, coplas y seguidillas [...] Posteriormente han ido tomando las referidas tonadillas tanto incremento que en el día son verdaderas piezas de música, o unas cortas escenas de ópera, algunas serias, y de una clase de música que pide mucho trabajo y meditación?.

Hemos de tomar en cuenta que, en la Península, la tonadilla se presentará no sólo como un mero intermedio de las comedias sino, además, como un intermedio compartido. Así, se hacía entre la primera y segunda jornada, compartiendo espacio con el entremés y, entre la segunda y la tercera, con el sainete. Poco a poco, sin embargo, al permitírsele ocupar un mayor espacio (el entremés será eliminado y el sainete pasado al final de la comedia) la tonadilla pasó a hacerse más larga y compleja. En Venezuela, la tonadilla nunca llegará a ocupar intermedio con el entremés (pues ésta entrará en escena cuando éste desaparece) pero sí con el sainete, con el que, poco a poco, dejará de compartir intermedio. A esto hemos de añadir que en Caracas, como ocurre en 1812, en algunas ocasiones la tonadilla deja de ser un mero intermedio para ser presentada de manera independiente, algo que, hasta donde conozco, no ocurrió en ningún otro sitio ${ }^{10}$.

Todo este proceso, tuvo que llevar, necesariamente, a que las tonadillas fueran cada vez más largas y explica el que, en las fuentes secas (mas nunca en las partituras), se les denomine habitualmente operetas. Pero ¿esta nueva denominación hacía referencia sólo a la longitud o también a un cambio en la estructura? A través del análisis de las 15 tonadillas escénicas que sabemos que fueron interpretadas en la Venezuela Colonial, hemos podido constatar que existía una clara diferencia morfológica entre lo que la prensa llama tonadilla y lo que denomina opereta y que, como Laserna ya 1-300.

9 Biblioteca Nacional de España, Madrid (BNE), Mss/14076

10 CAPELÁN (2015): vol. 1, 337-341. 
indicaba en 1792 , se trataban de verdaderas óperas cortas. De este modo hemos podido clasificar el repertorio denominado en las partituras como "tonadilla" del siguiente modo:

I. Tonadillas: son aquellas que tienen una estructura tripartita y carecen de parolas $^{11}$. La división en tres partes puede ser de dos tipos: la más antigua formada por Entable Coplas - Seguidillas epilogales o la más moderna integrada por Entable - Coplas - Final.
I A Entable - Coplas - Seguidillas: este modelo está formado por un Entable de varios movimientos que incluye un recitativo. Coplas de seis estrofas con dos sentencias en otra versificación y unas seguidillas epilogales con un tema literario completamente ajeno al de la tonadilla ${ }^{12}$.

A este esquema pertenece la obra de Laserna Los frenos trocados la cual, al llegar a Venezuela sufre una transformación al añadirle un compositor local un final de moraleja.

\begin{tabular}{|c|c|c|c|c|}
\hline \multirow{12}{*}{ Entable } & \multicolumn{4}{|c|}{ Laserna. Los frenos trocados } \\
\hline & \multicolumn{4}{|c|}{ I. $(\mathrm{Eb})$ para $S$, ob 1 y 2 , tpa 1 y 2 , vl 1 y 2, b, $2 / 4$} \\
\hline & $\begin{array}{c}\mathrm{A} \\
\left.\text { (cc. } 39^{2}-47\right)\end{array}$ & $\begin{array}{c}\text { B } \\
\left.\text { (cc. } 48^{2}-61^{1}\right)\end{array}$ & & \\
\hline & \multicolumn{4}{|c|}{ II. $(\mathrm{Bb})$ para $\mathrm{S}$, ob 1 y 2 , tpa 1 y 2 , vl 1 y $2, \mathrm{~b}, 3 / 8$} \\
\hline & $\begin{array}{c}\mathrm{A} \\
\text { (cc. 10-261) }\end{array}$ & $\begin{array}{c}\mathrm{B} \\
\left.\text { (cc. } 26^{1}-45^{1}\right) \\
\end{array}$ & $\begin{array}{c}\mathrm{A} \\
\left.\text { (cc. } 45^{1}-59^{1}\right)\end{array}$ & \\
\hline & \multicolumn{4}{|c|}{ III. (G) para $S$, ob 1 y 2 , tpa 1 y 2 , vl 1 y 2, b, 6/8 } \\
\hline & $\begin{array}{c}\mathrm{A} \\
\left.\text { (cc. } 4^{2}-12^{1}\right) \\
\end{array}$ & $\begin{array}{c}\mathrm{B} \\
\left.\text { (cc. } 13^{1}-20^{1}\right) \\
\end{array}$ & $\begin{array}{c}\mathrm{C} \\
\left.\text { (cc. } 20^{2}-27^{1}\right) \\
\end{array}$ & \\
\hline & $\begin{array}{|ll|}\mathrm{a} & \mathrm{a} \\
\end{array}$ & b b & & \\
\hline & \multicolumn{4}{|c|}{ Recitativo. (G) para $S$, vl 1 y 2, b, $4 / 4$} \\
\hline & \multicolumn{4}{|c|}{ IV. (F) para $S$, ob 1 y 2 , tpa 1 y 2 , vl 1 y 2, b, $2 / 4$} \\
\hline & $\begin{array}{c}\mathrm{A} \\
\left(\mathrm{cc} .8^{2}-21^{1}\right) \\
\end{array}$ & $\begin{array}{c}\mathrm{B} \\
\left.\text { (cc. } 21^{1}-28^{2}\right) \\
\end{array}$ & $\begin{array}{c}\mathrm{C} \\
\text { (cc. 291-36') }\end{array}$ & \\
\hline & & $\mathrm{b} \quad \mathrm{b}^{\prime}$ & $\mathrm{c} \mathrm{c}^{\prime}$ & \\
\hline \multirow[t]{3}{*}{ Coplas } & \multicolumn{4}{|c|}{ V. $(\mathrm{Bb})$ para $S$, ob 1 y 2, tpa 1 y 2, vl 1 y 2, b, $6 / 8,3 / 8$} \\
\hline & \begin{tabular}{|l}
{$[[: A$} \\
$\left(\right.$ cc. $\left.8^{2}-36^{2}\right)$ \\
\end{tabular} & $\begin{array}{c}\mathrm{A} \\
\left.\text { (cc. } 36^{2}-63^{2}\right) \\
\end{array}$ & $\begin{array}{c}\mathrm{A} \\
\left(\mathrm{cc} .63^{2}-91^{2}\right) \\
\end{array}$ & $\begin{array}{c}\mathrm{B}:]] \\
\text { (cc. } 92^{1}-136^{1} \text { ) }\end{array}$ \\
\hline & $\mathrm{a} \quad \mathrm{b} \quad \mathrm{c}$ & $\mathrm{a} \quad \mathrm{b} \quad \mathrm{c}^{\prime}$ & $\mathrm{a} \mathrm{b} \mathrm{c"}$ & $\mathrm{ddefgf}$ \\
\hline \multirow[t]{3}{*}{ Seguidillas } & \multicolumn{4}{|c|}{ VI. (F) para $S$, ob 1 y 2, tpa 1 y 2 , vl 1 y 2, b, 3/4, $2 / 4,3 / 4$} \\
\hline & $\begin{array}{c}\mathrm{A} \\
\left(\text { cc. } 7^{2}-26^{3}\right)\end{array}$ & $\begin{array}{c}\mathrm{B} \\
\left(\text { cc. } 27^{1}-100^{2}\right)\end{array}$ & $\begin{array}{c}\mathrm{A}^{\prime} \\
\left.\text { (cc. } 100^{2}-104^{1}\right)\end{array}$ & \\
\hline & \begin{tabular}{|ll}
$\mathrm{a}[[: \mathrm{b}$ & $\mathrm{c}$ \\
\end{tabular} & \begin{tabular}{|c|}
$\mathrm{d} \quad \mathrm{e}$ \\
\end{tabular} & $\mathrm{a}:]]$ & \\
\hline \multirow[t]{3}{*}{ Final } & \multicolumn{4}{|c|}{ VII. (F) para SS, vl 1y 2, b, 3/4 } \\
\hline & $\begin{array}{c}\mathrm{A} \\
\left(\text { cc. } 1^{3}-6^{2}\right) \\
\end{array}$ & $\begin{array}{c}\mathrm{B} \\
\left(\mathrm{cc} .7^{1}-10^{2}\right) \\
\end{array}$ & & \\
\hline & $\mathrm{a} \mathrm{a}^{\prime}$ & $\mathrm{b} \quad \mathrm{b}^{\prime}$ & & \\
\hline
\end{tabular}

Tabla 2. Morfología de la tonadilla Los frenos trocados de Laserna, conservada en Venezuela

11 En las tonadillas escénicas se denominaba parolas a las partes que se hacían habladas, sin ningún acompañamiento musical.

12 Existió también el modelo que sustituía las seguidillas epilogales por una tirana. No lo incluimos aquí porque ninguna de las tonadi- llas que sabemos que se interpretaron en la Venezuela colonial tenía este formato, que sí encontramos, sin embargo, en alguna de las tonadillas republicanas, por lo que seguramente también se utilizó en la época colonial, aún cuando actualmente no conservemos ningún ejemplo. 
El Entable, sección en la que se hace la captatio al público, está formado por cuatro movimientos que funcionan como uno solo mediante el atacca, en el tercero de los cuales encontramos un corto recitativo seco.

Las Coplas poseen un modelo fijo tanto desde el punto de vista literario como musical: tres coplas que terminan en una estrofa con estructura de seguidilla, cuyo modelo completo se repite. Todas las coplas (A) tienen la misma estructura tripartita $(\mathrm{a} b \mathrm{c}$ ) y en la estrofa de seguidilla (B) encontramos el esquema musical típico de este género (f $\mathrm{g}$ f) sólo a partir del cambio de compás a $3 / 8$ con lo que apreciamos que la forma literaria y la musical de seguidilla sólo calzan a partir de este cambio. Las Seguidillas tienen una estructura tripartita (A B A') en la que B se diferencia claramente por el cambio a un compás binario y por poseer figuras de mayor duración.

Para cuando esta tonadilla se representa en Caracas, ya se había comenzado el movimiento neoclásico en la ciudad que abogaba porque sólo se representasen aquellas obras en las que se respetara la unidad de acción. Por ello, una obra en la que se incluían unas seguidillas que nada tenían que ver el tema argumental de los dos movimientos anteriores, estaba en franca confrontación con las órdenes gubernamentales. Debió ser ésta la razón que llevó a un compositor local a añadir un Final, en el que se incluía como moraleja una alabanza a la virtud. Con ello no sólo se dejaba contentas a las autoridades sino que, también, se "corregía" un modelo tripartito que para el momento debía considerarse ya anticuado.

I B Entable - Coplas - Final: un Entable formado por varios movimientos, unas Coplas constituidas por seis estrofas más dos sentencias en otra versificación y un Final de moraleja en versos heptasílabos.

A este esquema pertenecen las tonadillas de Blas de Laserna La viuda de seis maridos, Mas no quiero murmurar y La civilización cuyas estructuras podemos apreciar en el siguiente cuadro:

\begin{tabular}{|c|c|c|c|c|c|c|c|}
\hline \multirow{13}{*}{ Entable } & \multicolumn{3}{|c|}{ La viuda de seis maridos } & \multicolumn{2}{|c|}{ Mas no quiero murmurar } & \multicolumn{2}{|c|}{ La civilización } \\
\hline & \multicolumn{3}{|c|}{$\begin{array}{l}\text { I. (g) para } S, \text { ob } 1 \text { y } 2, \mathrm{cl}, \text { fg, vl } \\
1 \text { y } 2 \text {, vla, b, } 2 / 4\end{array}$} & \multicolumn{2}{|c|}{$\begin{array}{l}\text { I. (C) para } S \text { ob } 1 \text { y } 2 \text {, tpa } 1 \text { y } 2 \text {, vl } \\
1 \text { y } 2, b, 4 / 4\end{array}$} & \multicolumn{2}{|c|}{$\begin{array}{l}\text { I. (Bb) para } S \text {, ob } 1 \text { y } 2, \text { tpa } 1 \text { y } \\
2, \text { vl } 1 \text { y } 2, b, 4 / 4\end{array}$} \\
\hline & A & B & C & A & $B$ & A & B \\
\hline & $\mathrm{a} \mathrm{a}^{\prime} \mathrm{b}$ & $\mathrm{ccd}$ & e eff $g$ & $\mathrm{a}^{\prime} \quad \mathrm{a}^{\prime}$ & b $\quad c$ & $a a^{\prime} b$ & $\mathrm{c} \quad \mathrm{d}$ \\
\hline & & & & \multicolumn{2}{|c|}{$\begin{array}{l}\text { II. (a) para } S \text {, ob } 1 \text { y } 2 \text {, tpa } 1 \text { y } 2 \text {, } \\
\text { vl } 1 \text { y } 2, b, 6 / 8\end{array}$} & \multicolumn{2}{|c|}{$\begin{array}{l}\text { II. (g) para } S \text {, (ob } 1 \text { y } 2) \text { vl } 1 \text { y } \\
2, b, 3 / 4\end{array}$} \\
\hline & & & & A & $\mathrm{B}$ & \multirow[t]{2}{*}{ A } & \multirow{2}{*}{$\begin{array}{l}\text { B } \\
\text { boleras }\end{array}$} \\
\hline & & & & \multirow[t]{2}{*}{ a $\quad a^{\prime}$} & & & \\
\hline & & & & & & $\mathrm{b}$ & $\begin{array}{l}\text { boleras } \\
\text { c d c }\end{array}$ \\
\hline & \multicolumn{3}{|c|}{$\begin{array}{l}\text { II. Recitativo [acompañado] } \\
\text { (C) para S, fg, vl } 1 \text { y } 2 \text {, vla, b, } \\
4 / 4\end{array}$} & \multicolumn{2}{|c|}{$\begin{array}{l}\text { III. Recitativo [seco] (C) para S, } \\
\text { vl } 1 \text { y } 2, \text { b, } 4 / 4 \\
\text { Va atacca }\end{array}$} & \multicolumn{2}{|c|}{$\begin{array}{l}\text { III. (Eb) para } S \text {, ob } 1 \text { y } 2, \text { Tpa } 1 \\
\text { y } 2 \text {, vl } 1 \text { y } 2, \text { b, } 3 / 4 .\end{array}$} \\
\hline & \multirow{2}{*}{\multicolumn{3}{|c|}{$\begin{array}{l}\text { III. (F) para } S \text {, fg, vl } 1 \text { y } 2 \text {, vla, } \\
\text { b, } 6 / 8\end{array}$}} & \multirow{2}{*}{\multicolumn{2}{|c|}{$\begin{array}{l}\text { IV. (C) para S, ob } 1 \text { y } 2 \text {, tpa } 1 \text { y } 2 \text {, } \\
\text { vl } 1 \text { y } 2, b, 2 / 4\end{array}$}} & & \\
\hline & & & & & & \begin{tabular}{|l|}
$\mathrm{A}$ \\
\end{tabular} & $\mathrm{B}$ \\
\hline & A & $\mathrm{B}$ & $\mathrm{C}$ & $\mathrm{A}$ & B & \multirow[t]{2}{*}{$\begin{array}{|ll|}\mathrm{a} & \mathrm{a} \\
\end{array}$} & $\mathrm{b} c \mathrm{~d}$ \\
\hline & & $\mathrm{bb}^{\prime} \mathrm{c}$ & & $\mathrm{a} \quad \mathrm{a}$ & & & \\
\hline
\end{tabular}




\begin{tabular}{|c|c|c|c|c|c|c|c|c|c|c|c|c|}
\hline \multirow[t]{3}{*}{ Coplas } & \multicolumn{4}{|c|}{$\begin{array}{l}\text { IV. (D) para } S \text {, ob } 1 \text { y } 2, \mathrm{cl}, \mathrm{fg} \text {, } \\
\text { vl } 1 \text { y } 2 \text {, vla, b, } 3 / 4\end{array}$} & \multicolumn{4}{|c|}{$\begin{array}{l}\text { V. (G) para } S \text {, ob } 1 \text { y } 2 \text {, tpa } 1 \text { y } 2 \text {, } \\
\text { vl } 1 \text { y } 2, \text { b, } 2 / 4,3 / 4\end{array}$} & \multicolumn{4}{|c|}{$\begin{array}{l}\text { IV. (Bb) para } S \text {, ob } 1 \text { y } 2 \text {, tpa } 1 \text { y } \\
2 \text {, vl } 1 \text { y } 2, b, 2 / 4\end{array}$} \\
\hline & [[:A & A & A & $\begin{array}{c}\mathrm{B}:]] \\
\text { seguidilla }\end{array}$ & {$[[: \mathrm{A}$} & A & A & $\mid \begin{array}{c}\mathrm{B}:]] \\
\text { seguidillas }\end{array}$ & [[:A & A & $A$ & \begin{tabular}{|c|}
$\mathrm{B}:]]$ \\
seguidillas
\end{tabular} \\
\hline & $a b c$ & $\mathrm{abc}$ & $a b c$ & de de d & $a b c$ & $\mathrm{abc}$ & $a b c$ & deded & \begin{tabular}{|l}
$a b c$ \\
\end{tabular} & $a b c$ & $a b c$ & deded \\
\hline \multirow[t]{3}{*}{ Final } & \multicolumn{4}{|c|}{$\begin{array}{l}\text { V. (G) para } S, \text { ob } 1 \text { y } 2, \mathrm{cl}, \text { fg, vl } \\
1 \text { y } 2 \text {, vla, b, } 2 / 4\end{array}$} & \multicolumn{4}{|c|}{$\begin{array}{l}\text { VI. (Bb) para } S \text {, ob } 1 \text { y } 2 \text {, tpa } 1 \text { y } 2 \text {, } \\
\text { vl } 1 \text { y } 2, b, 2 / 4\end{array}$} & \multicolumn{4}{|c|}{$\begin{array}{l}\text { V. (G) para } S, \text { ob } 1 \text { y } 2, \text { tpa } 1 \text { y } 2 \text {, } \\
\text { vl } 1 \text { y } 2, b, 2 / 4\end{array}$} \\
\hline & \multicolumn{2}{|c|}{ A } & B & $A^{\prime}$ & A & & B & $A^{\prime}$ & A & & B & $A^{\prime}$ \\
\hline & \multicolumn{2}{|c|}{$a a^{\prime} b b_{c}$} & c e f & $\mathrm{a} \mathrm{a}^{\prime} \mathrm{g} \mathrm{g}^{\prime} \mathrm{g}^{\prime}$ & $a a^{\prime} b$ & & $c^{\prime} d$ & $a a^{\prime} b e$ & $a a^{\prime} b$ & & $c^{\prime} d$ & $\mathrm{a}^{\prime} \mathrm{b}^{\prime} \mathrm{e}$ \\
\hline
\end{tabular}

Tabla 3. Morfología de cada uno de los movimientos de las tonadillas tripartitas del modelo Entable - Coplas - Final

Como se aprecia en la tabla, de todas las secciones, el Entable es el único que no tenía una estructura preestablecida. Podía o no tener recitativo, su número de movimientos o cambios de compás era variable y la forma de éstos podía ser binaria o ternaria.

Las Coplas, en las que se presentaba el asunto central de la tonadilla, tenían una estructura prefijada, tanto en la forma general (letras mayúsculas) como en cada una de sus secciones (letras minúsculas) que ya encontramos en el formato tripartito arcaico que analizamos en Los frenos trocados. Se dividía en dos grandes partes formada cada una por tres coplas más una estrofa en otra versificación que tenía un función tanto textual (para dar una sentencia o moraleja de lo planteado) como musical (para dividir la obra). Esta última estrofa no era sino unas seguidillas, tanto desde el punto de vista literario como musical.

El último movimiento, denominado Final, era siempre de moraleja con una estructura prefijada tripartita (A B A'). No sólo la estructura, sino también las características musicales eran las de un aria. Si bien las dos primeras secciones (A y B) no poseen siempre un movimiento melódico cercano al aria, sí podemos encontrar las características de este género en $\mathrm{A}^{\prime}$. Con ello podemos decir que se amplía aquí la influencia de la música italiana (ya presente en los recitativos de los Entables) al último de los movimientos.
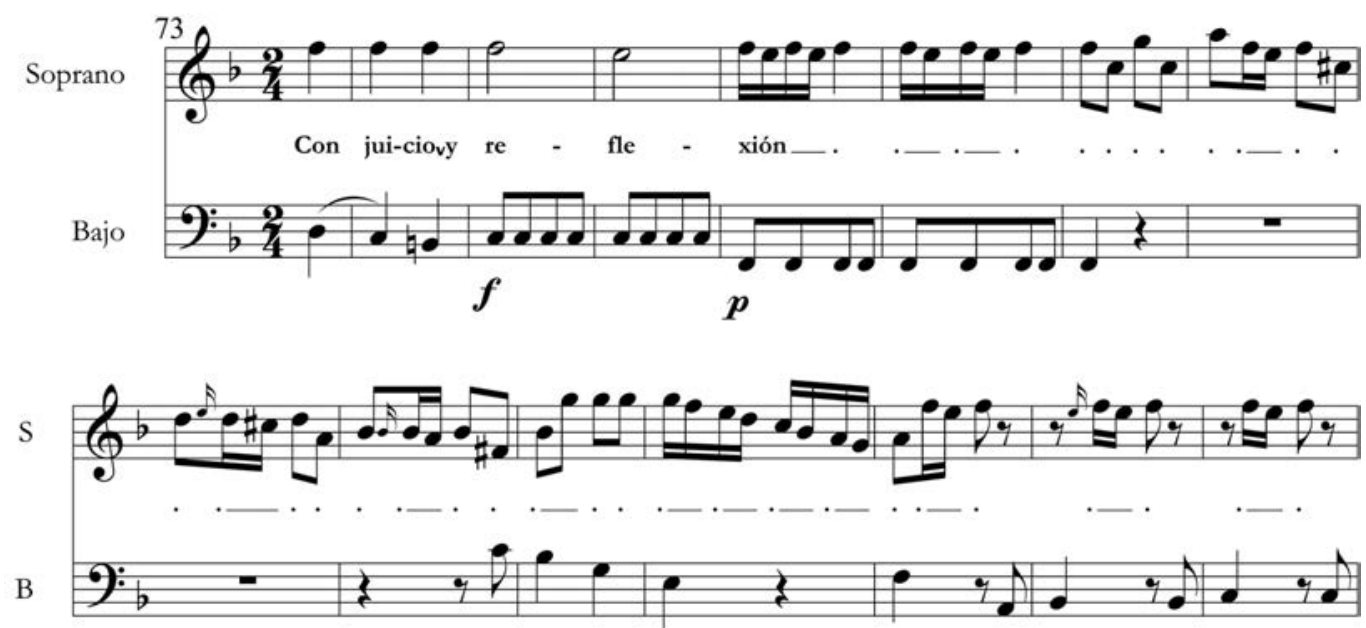

Figura 3. Guión del Final de La viuda de seis maridos de Laserna en el que se aprecia la línea melódica que le da el carácter de aria. 
II. Operetas: son aquellas que no tienen una forma fija (ni tripartita ni de otro tipo) carecen de entable, tienen una o varias parolas y si bien pueden tener secciones como las coplas, seguidillas o final, éstas ni son imprescindibles ni tienen (a excepción del final) un lugar fijo en la obra.

Entre las interpretadas en la Venezuela colonial tenemos los siguientes modelos:

a) Con 1 parola y recitativo: El amante rendido y $E l$ accidente fingido de Laserna.

b) La mitad de los movimientos con parola: El asturiano burlado de Laserna.

c) Todos los movimientos con parola excepto el antepenúltimo o el penúltimo y el último: El contrato matrimonial, El hombre mujer y El amor por poderes de Moral y $E l$ médico y sus sobrinos de Laserna.

d) Todos los movimientos con parola excepto el último: La italiana y la andaluza de Laserna ${ }^{13}$.

El discurso narrativo es mucho más complejo en las operetas para lo cual se sirven de la presencia de varios personajes y de un buen número de parolas que ayudan y ace- leran la acción. Esto hace que las coplas (lugar en que en el momento tripartito se narraba el argumento) ya no sean indispensables, si bien las seguimos encontrando en diferentes operetas. Sin embargo, ya no tendrán un número fijo de seis coplas y la parte B casi nunca está presente y, en el caso de estarlo, ya no es una seguidilla. Eso sí, A seguirá teniendo la forma tripartita $(a b c)$.

Los bailes son habituales en las operetas, encontrando más géneros de este tipo que en las tonadillas. Es usual la presencia de seguidillas, que ahora en ningún caso son epilogales, son de menor extensión y suelen contar con independencia melódica (es decir, cuando se vuelven a hacer los versos, la música no tiene por qué repetirse). También se incluyen boleras y tiranas.

En El amante rendido de Laserna se incluye una tirana a dúo, de estructura tripartita y en compás de 3/8. En el estribillo, encontramos las interjecciones y los desplazamientos métricos habituales en este tipo de bailes, elemento sobre el que volveremos al hablar de la tonadilla escénica en Venezuela en la época republicana.

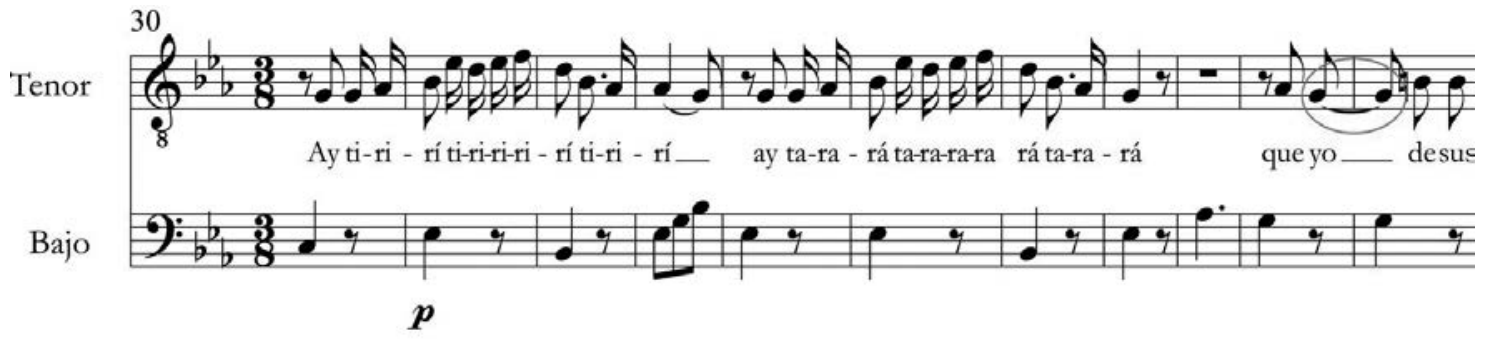

$\mathrm{T}$

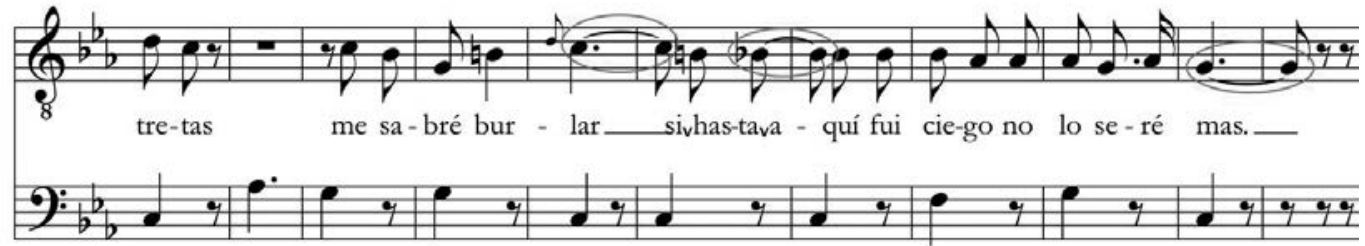

Figura 4. Tirana de El amante rendido de Laserna en el que se aprecian las interjecciones y los desplazamientos métricos

13 Queda fuera de este análisis la tonadilla El enemigo de las mujeres de Laserna por no calzar con ninguno de los esquemas propuestos estando cercana a lo que Subirá denominó tonadillas de pasos. 
El último movimiento de las operetas no es ya un "final de moraleja" sino un "final con moraleja", es decir, movimientos que sólo en la última parte tienen este tipo de sentencias. Pero lo más destacable, en todo caso, es que su forma musical no es ya, en ningún caso, la de un Aria da Capo.

Podemos apreciar así que el paso de la tonadilla a la opereta no constituyó una italianización del género, como asegura José Subirá (1928, 206-207) sino, muy por el contrario, de hispanización. Los recitativos terminarán desapareciendo ante la introducción cada vez mayor de parolas y los Finales, que no eran sino arias da capo en las tonadillas, en las operetas terminarán siendo sustituidos por movimientos que ya no tienen esta forma. Se da también una irrupción de bailes castizos como la tirana, la seguidilla o las boleras. Ciertamente, las seguidillas eran una parte esencial del primer modelo de tonadilla tripartita, pero habían casi desaparecido cuando, en el segundo modelo tripartito, son sustituidas por finales en forma de aria.

\section{LA TONADILLA ESCÉNICA DURANTE LA ÉPOCA REPUBLICANA O EL PROCESO DE CRIOLLIZACIÓN DE UN GÉNERO CASTIZO}

Bien conocida es la división que hace José Subirá de la tonadilla escénica: Aparición y albores (1751-1757), Crecimiento y juventud (1757-1770), Madurez y apogeo (17711790), Hipertrofia y decrepitud (1790-1810), Ocaso y olvido (1811-1845) (Subirá 1928, I, 97, 117, 157, 205, 241) ${ }^{14}$. Esta periodización, sin embargo, es inaplicable en Venezuela, en donde el denominado período de decrepitud fue uno de los momentos con que mejor salud contó la tonadilla y en los años siguientes, una vez conseguida la independencia, no sólo no fue olvidada, sino interpretada con asiduidad. Esta pervivencia en el tiempo fue posible debido a un proceso de actualización del género que se logró, en gran parte, gracias al proceso de criollización de la tonadilla escénica que se da en Venezuela.

Muestra de la persistencia del género una vez conseguida la independencia son los documentos conservados. Así tenemos que en 1823, por ejemplo, el Ayuntamiento de Caracas, ante la puesta en escena de una tragedia que consideran inmoral, acuerda que en lo sucesivo no debe presentarse ninguna comedia ni tonadilla antes de pasar la censura municipal ${ }^{15}$. La prohibición se repite un año después ${ }^{16} \mathrm{y}$ se reitera en el reglamento de teatro del 19 de enero de 1829 en el que, entre las funciones del censor está la de examinar las comedias, tragedias, tonadillas, sainetes y cualesquiera otras piezas dramáticas ${ }^{17}$.

No fue Caracas el único sitio en el que se siguieron interpretando tonadillas. En 1843 el español Francisco Villalba presenta en Cumaná La venida del soldado a la que la prensa llama la "usada y fastidiosa tonadilla", lo que muestra que su interpretación era más que habitual ${ }^{18}$. Llegaron a existir, incluso, interpretaciones de tonadillas realizadas por niños como deja ver un manuscrito conservado en la Biblioteca Nacional de Venezuela (Jal 1013) en el que se copia la tonadilla Los cazadores y la paya con indicación de haber sido transportada para que éstos pudieran cantarla. La obra no es otra que La paya y los cazadores de Pablo Esteve, en copia del venezolano Atanacio Bello Montero, quien a partir de 1834, establece una compañía teatral con la que interpretaba, entre otro repertorio, tonadillas escénicas ${ }^{19}$.
14 Esta periodización, en los últimos años, ha sido puesta en entredicho incluso para el caso Peninsular. Véase LOLO, XXV, 2 (Madrid, 2002): 465 y LE GUIN (2008): 183-223.
15 AZPARREN (1997): 41.

16 Actas y documentos que contienen las disposiciones acordadas para el examen previo de las piezas dramáticas que se representen en el teatro de esta capital desde su restablecimiento hasta el presente. Caracas, Folleto impreso, 1837, pp. 2-3.

17 Íbid, p. 12.

18 GARCÍA (2006): 49. Debe ésta tratarse de la tonadilla que, bajo este título, compuso Blas de Laserna y que actualmente se conserva en la Biblioteca Histórica de Madrid bajo la signatura Mus 188-6. Esta obra fue estrenada en 1805 y puesta otra vez en escena en 1808 en Madrid. En 1811 se presenta en Cádiz. En 1810 la obra también fue presentada en Cuba ("Teatros". Diario de Madrid, 61, 1/3/1808, p. 272; ROMERO (2009): 329-343; VILLABELLA).

19 OJEDA Y CAPELÁN (2012): 31-42. 


\begin{tabular}{|c|c|c|c|}
\hline AUTOR & TÍTULO & FUENTE & OBSERVACIONES \\
\hline Pablo Esteve & Los cazadores y la paya & AABN Jal 1013 & $\begin{array}{l}\text { Copia de Atanacio Bello, } \\
\text { transportada para ser interpretada } \\
\text { por niños. }\end{array}$ \\
\hline Atanacio Bello & La inocente serranilla & AABN Jal 1580, 2000, 2415 & $\begin{array}{l}\text { Mismo texto de la obra homónima } \\
\text { de Blas de Laserna, música (la } \\
\text { mayor parte) distinta }\end{array}$ \\
\hline José Ángel Montero & La inocente serranilla & AABN CBM 2213 & $\begin{array}{l}\text { Texto en gran parte equivalente } \\
\text { a la obra homónima de Laserna. } \\
\text { Música distinta. }\end{array}$ \\
\hline ¿Blas de Laserna? & La venida del soldado ${ }^{20}$ & $\begin{array}{l}\text { García, Sonia. Teatro y } \\
\text { música en Cumaná. Siglo } \\
\text { XIX, p. } 49\end{array}$ & $\begin{array}{l}\text { No se conserva ni la partitura ni el } \\
\text { libreto en Venezuela, sino sólo una } \\
\text { referencia en la prensa, por lo que } \\
\text { es imposible saber si es la obra } \\
\text { homónima de Laserna. }\end{array}$ \\
\hline José Ángel Montero & Los majos de rumbo & $\begin{array}{l}\text { AABN, Manuscritos sin } \\
\text { ingreso en la base de datos } \\
\text { y CAJ 1079 }\end{array}$ & $\begin{array}{l}\text { A pesar del título homónimo, se } \\
\text { trata de una obra completamente } \\
\text { diferente a la de Jacinto Valledor }\end{array}$ \\
\hline José Ángel Montero & La pepita & BNV MSV Cat 1803 & $\begin{array}{l}\begin{array}{l}\text { Sólo se ha podido localizar el } \\
\text { libreto }\end{array} \\
\end{array}$ \\
\hline $\begin{array}{l}\text { José Ángel Montero / Pablo } \\
\text { del Moral }\end{array}$ & El desafio del abanico & BNV MSV Cat 1803 & $\begin{array}{l}\text { Sólo se conserva el libreto, } \\
\text { que es coincidente con la obra } \\
\text { homónima del español Pablo del } \\
\text { Moral }^{21} \text {. La música, sin localizar, } \\
\text { es muy probable que fuera de J. A. } \\
\text { Montero. }\end{array}$ \\
\hline José Ángel Montero & La curiosidad de las mujeres & AABN Jal 2003 & $\begin{array}{l}\text { Es el mismo texto (con adaptación } \\
\text { de J. J. Alcoita) de la tonadilla } \\
\text { homónima de Laserna. Música } \\
\text { distinta. }\end{array}$ \\
\hline $\begin{array}{l}\text { Mariano Fernández (texto)/ } \\
\text { ¿J.A. Montero o S. Fuertes? }\end{array}$ & La castañera & BNV MSV CBK 9404 & $\begin{array}{l}\text { Sólo se conserva el libreto que es } \\
\text { coincidente con la obra homónima } \\
\text { de Mariano Fernández (texto), } \\
\text { musicalizada por Soriano Fuertes. } \\
\text { Desconocemos si J. A. Montero } \\
\text { realizó una nueva música para esta } \\
\text { obra }^{22} \text {. }\end{array}$ \\
\hline
\end{tabular}

Tabla 2. Tonadillas escénicas interpretadas en Venezuela durante la época republicana

20 Existe un Juguete lírico-dramático de José Ángel Montero de título similar, El regreso del soldado (AABN Jal 1027). A pesar de la similitud del título se trata de una obra completamente diferente a la de Laserna tanto musical como literariamente. En el caso venezolano se hace referencia a la guerra federal desarrollada en Venezuela entre 1859 y 1863.

21 La obra de Pablo del Moral se conserva en la Biblioteca Histórica de Madrid (BHM), la música bajo la signatura Mus 133-8 y el texto en Tea 220-130. El libreto de la versión madrileña está incompleto mientras que la venezolana está completa.

22 En los manuscritos venezolanos, El desafío del abanico aparece indicada como opereta y La inocente serranilla de J. A. Montero, La curiosidad de las mujeres y La castañera como zarzuelas. Los incluimos en este cuadro, sin embargo, porque tomaron los textos de tonadillas escénicas peninsulares. 
Si bien en la época republicana encontramos todavía la representación de tonadillas de autores peninsulares, lo cierto es que ya tenemos producción local de este género por parte de autores como Atanacio Bello Montero (1800-1876) o su primo José Ángel Montero (1832-1881) ${ }^{23}$. El hecho de que compositores caraqueños se dedicaran a la composición de este género en época tan tardía, no sólo llevará a la necesaria actualización de la tonadilla escénica, sino también a su criollización.

Un caso curioso es el de La inocente serranilla. En Venezuela tanto Atanacio Bello en 1835 como José Ángel Montero en 1865 tomarán el texto de la obra homónima de Blas de Laserna, pero cada uno de ellos le hará una música nueva. La transformación de esta tonadilla es mucho más amplia en el caso de José Ángel Montero, en la que encontramos importantes modificaciones del texto, y en la que hace una obra musical completamente nueva en la que introduce uno de los bailes que para ese momento estaba de moda: el vals.
El vals le servirá a J. A. Montero como un elemento unificador de toda la obra introduciéndolo como parte de cada uno de los tres movimientos de su composición. Ahora bien, ¿el autor caraqueño escribe valses a la europea o los típicos valses venezolanos?

Como es sabido, el vals, al llegar a Venezuela sufrirá algunas modificaciones. Entre las más importantes tenemos el cambio en el acompañamiento: de tres negras $(\downarrow\rfloor J$ ) se pasa a una negra, silencio de corchea, corchea y negra $(\downarrow, \lessgtr\rfloor)$. Otro elemento importante es la "melodía saltaperico", es decir, una línea melódica en la que se introduce un buen número de silencios e interrupciones ${ }^{24}$. Si bien este último elemento lo encontramos escuetamente en el primer movimiento de esta obra, lo cierto, es que el acompañamiento es el típico a la europea.

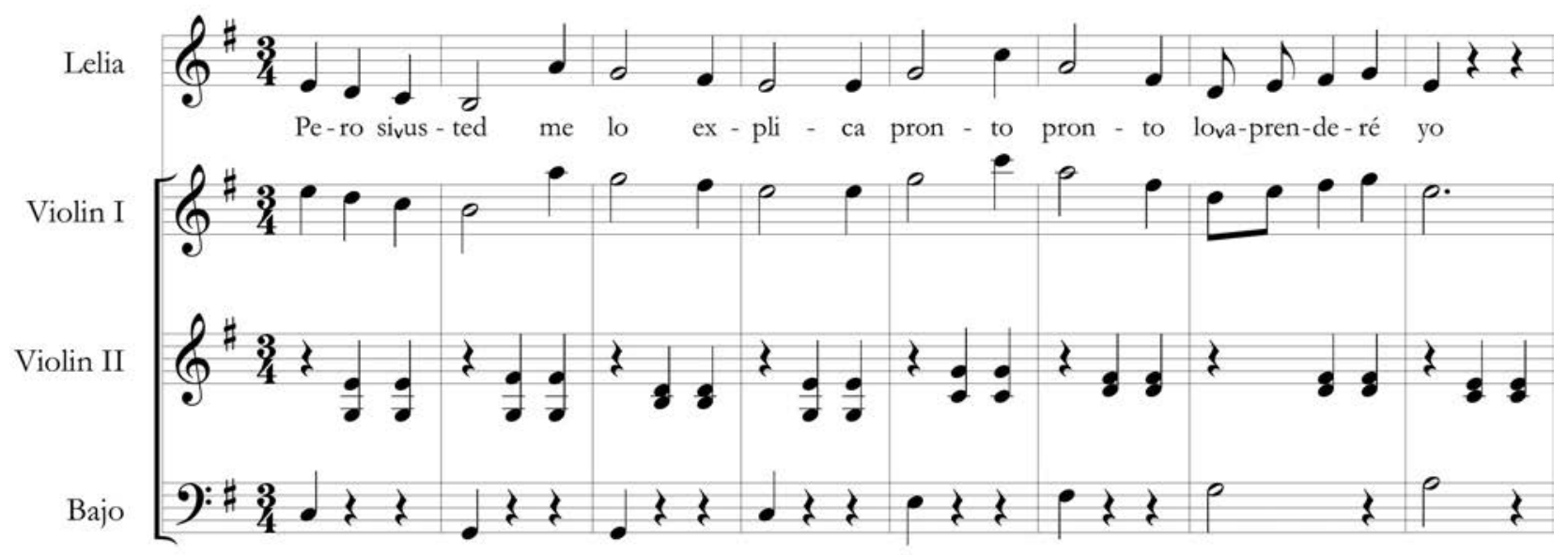

Figura 5. Segundo vals del primer movimiento de La inocente serranilla de J. A. Montero, en donde se puede a apreciar que se trata de un vals a la europea.

23 Alberto Calzavara (1987: 96) asegura que, durante la época colonial, Juan Bautista Olivares (1765-¿?), había sido compositor de tonadillas escénicas. Discrepamos, sin embargo de este autor, al considerar que su aseveración se trata de un error de interpretación en un documento en el que Olivares indica que puso los papeles de las tonadillas interpretadas en 1793 en Guatire, aseveración que nosotros entendemos como que las particellas eran de su propiedad, pero no necesariamente compuestas por él.
24 A este respecto véase el interesante artículo de PALACIOS, 35 (Caracas, 1997): 99-116. Tampoco hay cambios del compás de 3/4 al $6 / 8$ ni encontramos el ritmo $\perp 5$ I S que, como expone Juan Francisco Sans, están en alguno de los valses del Cuaderno de piezas de baile de Pablo Hilario Giménez (SANS, 2012: 355). 
Ahora bien, al analizar la obra nos queda abierta la pregunta si la criollización, no plasmada en el papel, se llevaría a cabo a la hora de la interpretación, modificando los segundos violines la primera de sus negras por un silencio de corchea y corchea y realizando el cantante cortes en la línea melódica.

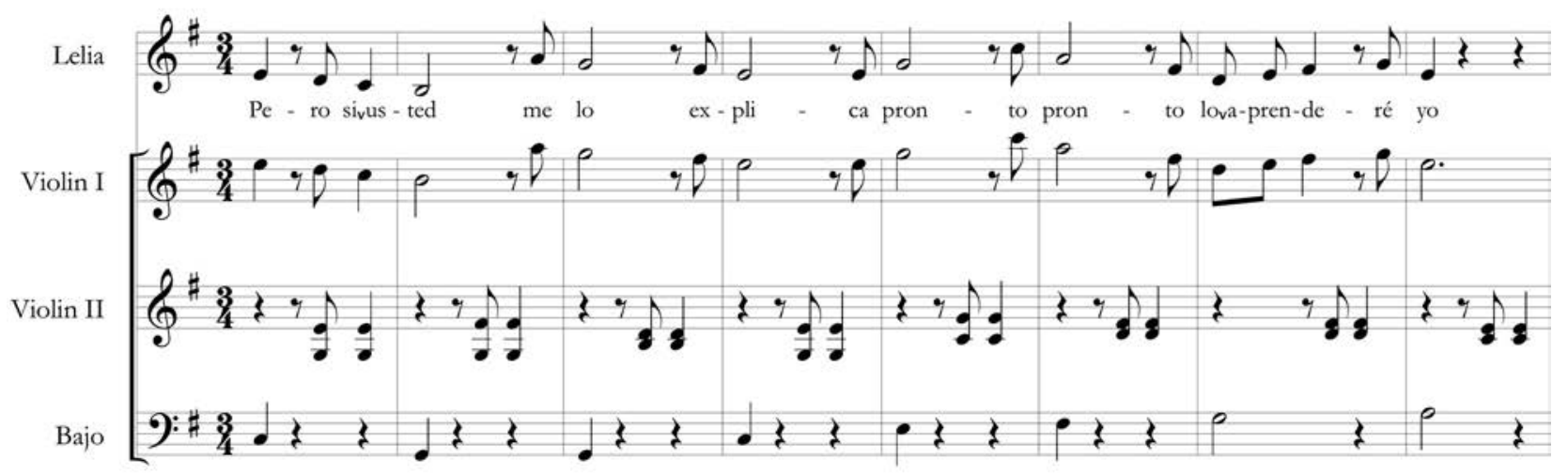

Figura 6. Propuesta de cómo podía ser realmente interpretado este vals de manera criollizada.

\subsection{De lo castizo a lo criollo: la tirana española o la ante- cesora del merengue americano}

Más allá de la hipótesis de la interpretación de valses a la venezolana en La inocente serranilla de J. A. Montero, podemos decir que encontramos un claro ejemplo de criollización en la tonadilla escénica Los majos de rumbo del mismo autor, en la que incluye varios merengues. Esta obra se conserva en la Biblioteca Nacional de Venezuela en dos versiones. La que denominaremos versión $A$, que se trata de una copia de Ramón Montero ${ }^{25}$, y la versión B, que tiene indicación de pertenecer a José Ángel Montero quien seguramente también fue el compositor ${ }^{26}$. Un análisis compara- tivo entre la versión A y la versión B de esta obra de José Ángel Montero, nos deja interesantes conclusiones. Más allá de las pequeñas diferencias entre una y otra (mayor extensión de las introducciones y de algunas frases, variaciones melódicas o distintas tonalidades) nos importa destacar la diferencia de escritura del ritmo en las danzas-merengue de la versión A y la versión B. Mientras en la primera el ritmo básico $(3+2)$ se escribe con una corchea dos semicorcheas y dos corcheas, en la segunda se hace con un tresillo de corchea y dos corcheas ${ }^{27}$.
25 AABN, Manuscritos musicales sin ingreso en la base de datos.

26 AABN, CAJ 1079
27 Fuera de la danza-merengue es de sumo interés el artículo de PESSARRODONA, 28 (Madrid, 2015). Esta autora identificó la presencia constante de lo que ella denomina también una célula $3+2$ en varias tonadillas de Jacinto Valledor. Si bien la manera de escribirla es diferente a la de los merengues venezolanos, es importante constatar su existencia en numerosas tonadillas españolas. Esta autora vincula estos ritmos de $3+2$ con la base del ritmo de tango y, según ella, más que una función de baile tendrían una finalidad dramatúrgico-musical. 


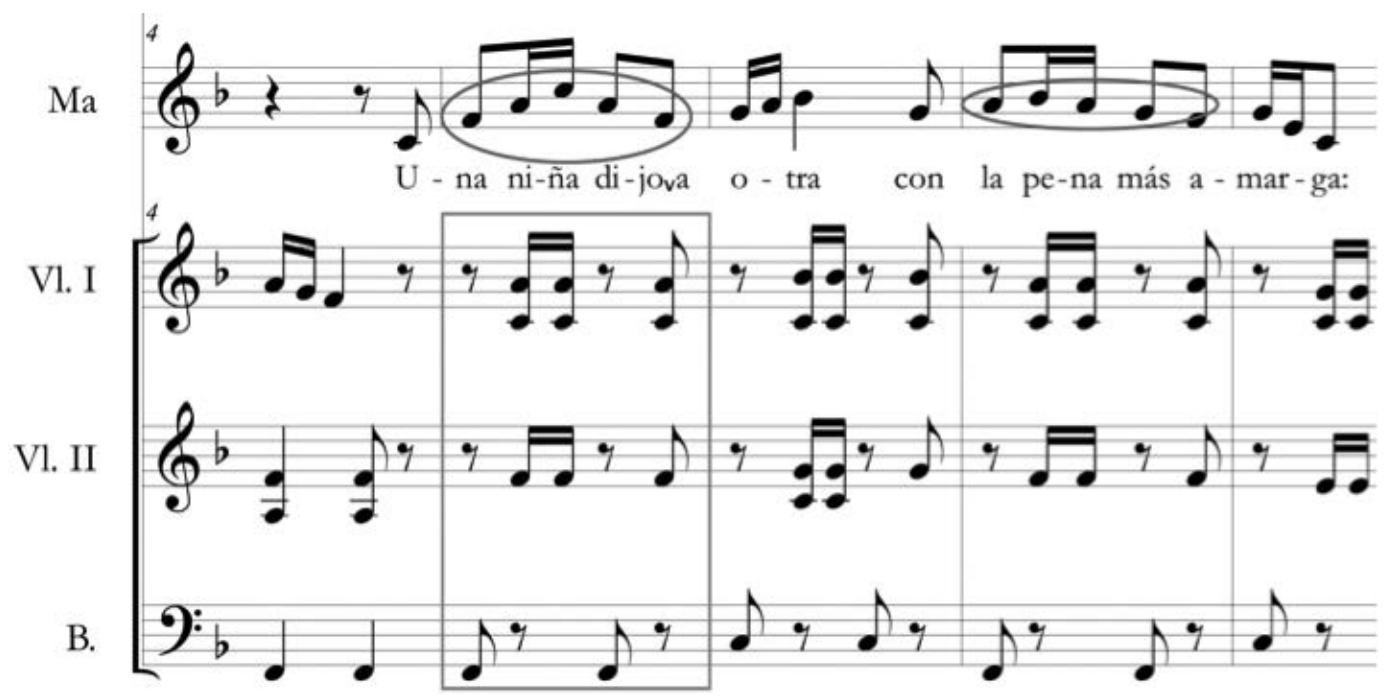

Figura 7. Último movimiento de Los majos de rumbo de J. A. Montero (versión A)

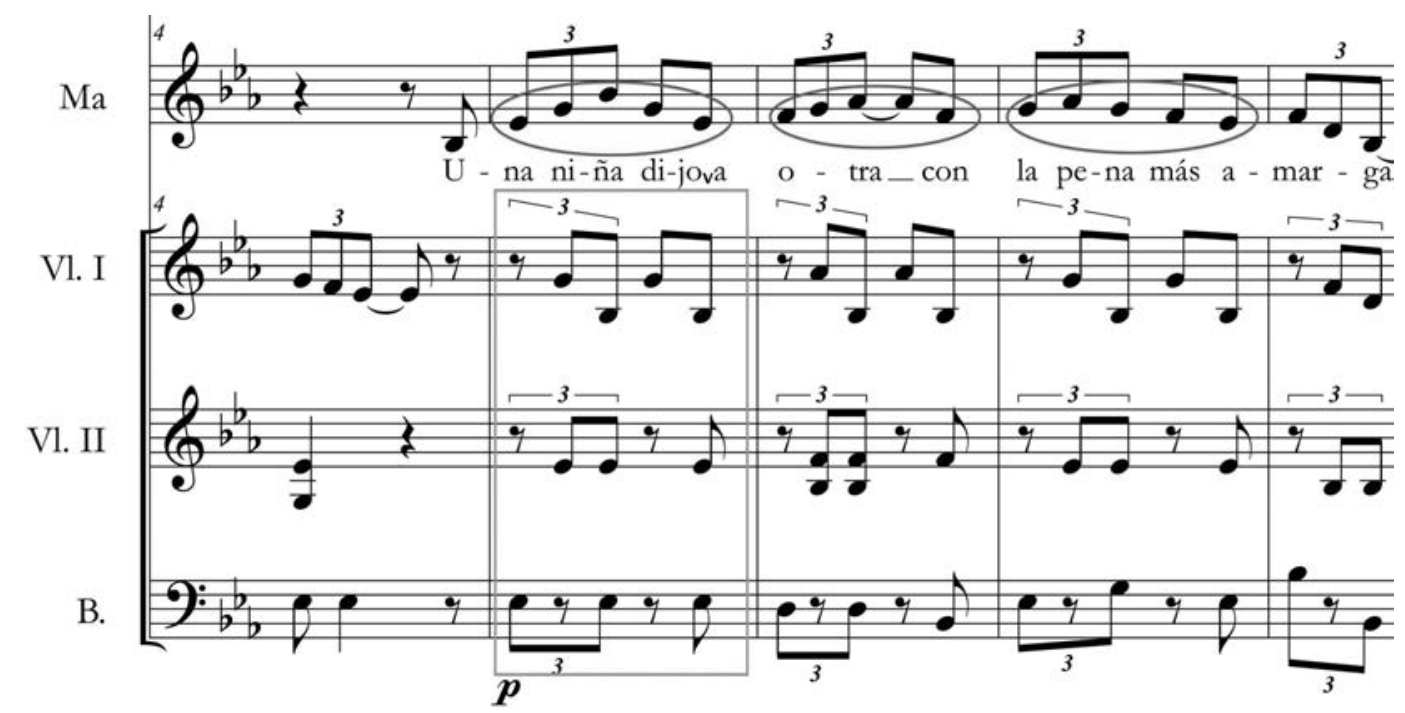

Figura 8. Último movimiento de Los majos de Rumbo de J. A. Montero (versión B) en el que se puede apreciar las diferentes maneras de escribir el $3+2$

Me atrevo a asegurar que la diferencia entre ambas no denota un cambio en la interpretación de la música sino una variación en el modo de escribir un mismo ritmo que, por popular, era de difícil plasmación en el papel para los compositores. Sería la versión B la que tendría una mayor cercanía con lo que realmente ocurría en la ejecución de las danzas-merengue.
Este movimiento final de la tonadillas Los majos de rumbo de J. A. Montero es de sumo interés para el estudio del merengue, no sólo porque nos muestra dos maneras de escribir la melodía que convivían sino, principalmente, porque nos ha permitido llegar a establecer una propuesta sobre cuál es el origen del merengue. 
Juan Francisco Sans, en su tesis doctoral, Los bailes de salón en la Venezuela del siglo XIX: géneros, interpretación y estilo, se hace eco de que el origen de la danzamerengue está en la contradanza ${ }^{28}$. No negaremos aquí esta relación, mucho más cuando los ejemplos 7 y 8 puestos más arriba, nos parecen una muestra de ella: en el ejemplo 7 el acompañamiento no es otro que el típico de las contradanzas (con la corchea sustituida por dos semicorcheas en el primer tiempo) que poco a poco será siendo modificado hasta el figuraje presente en el ejemplo 8 , que no es otro que el típico acompañamiento de merengue, que ya establecía Heraclio Fernández en su Nuevo método para aprender a acompañar en el piano toda clase de piezas y en especial las de baile al estilo venezolano en 1876 (reeditado en 1883).
Sin embargo, considero que existe otro antecedente del merengue que, hasta donde sé, no ha sido establecido por ningún otro autor: la tirana. Mi investigación a este respecto comenzó, justamente, con el estudio del último movimiento de Los majos de rumbo de J. A. Montero. Musicalmente es claramente un merengue pero literariamente, consiste en una tirana: cuartetas octosílabas con una rima asonante los pares y un estribillo con palabas sonaras inventadas.

Este hecho me llevó a investigar entre las numerosas tonadillas escénicas españolas en las que hay tiranas. Pude encontrar así una tonadilla española, Los maestros de la Raboso, cuyo estribillo de la tirana coincidía tanto textual como melódicamente con la de J. A. Montero ${ }^{29}$.

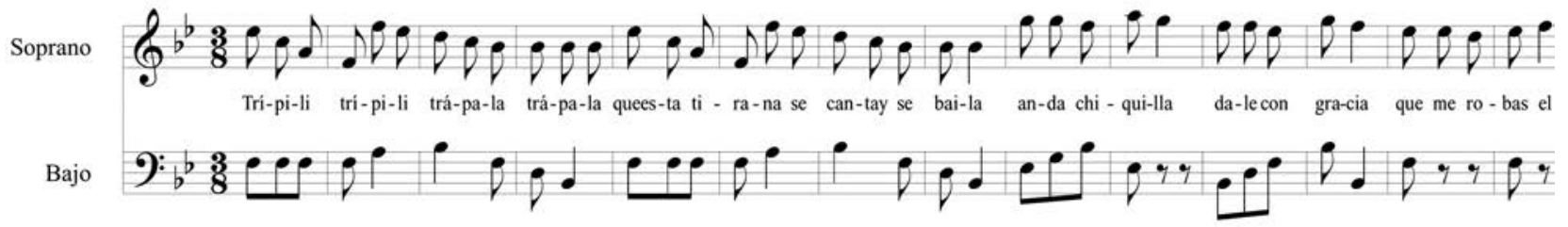

Figura 9. Estribillo de la Tirana de Trípili de Pablo Esteve (BHM, Mus 188-7)

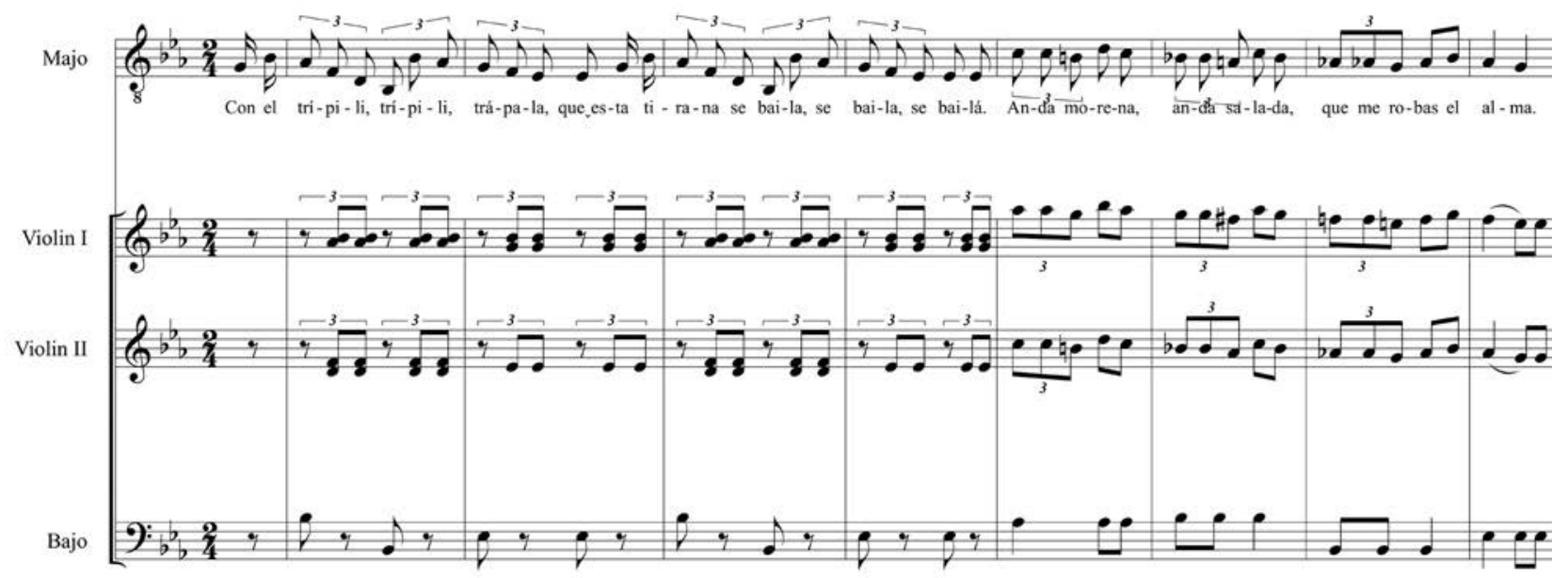

Figura 10. Merengue final de Los majos de rumbo de J. A. Montero (AABN CAJ 1079)

29 Se trata de una tonadilla del mismo título, pero completamente distinta, a la de Ramón Carnicer que fue editada por José Subirá. Tanto esta obra como la de Carnicer podemos encontrarla en la Biblioteca Histórica de Madrid, bajo la signatura Mus 188-7. La tirana no es otra que la Tirana de Trípili, la cual fue musicalizada por diferentes autores. 
Como se puede apreciar comparando los dos fragmentos, se trata de la misma melodía pero binarizada. Es decir, con la simple conversión de una tirana en compás ternario a un compás binario, J. A. Montero consigue un merengue convencional. Cabe decir, además, que los desplazamientos métricos propios de la tirana, son también una característica del merengue en donde el no "hacer tierra" (no caer en la primera parte de un tempo) es una de sus características, muy probablemente heredadas de las tiranas.

Con ello podemos ahora decir que al proceso de binarización del tempo que se da en Latinoamérica (del que nos hablan Rolando Pérez Fernández y Faustino Núñez ${ }^{30}$ ) hay que añadirle ahora el proceso de binarización del compás. No encontraríamos así, únicamente el paso de un 6/8 al 2/4 como ocurre en la contradanza sino, también, el paso del 3/4 al $2 / 4$ que ocurre en la tirana y que la termina convirtiendo en el merengue ${ }^{31}$.

\section{CONCLUSIONES}

José Subirá plantea la italianización de la tonadilla como la causa de su desaparición ${ }^{32}$. Con el corpus de obras interpretadas en la Venezuela colonial analizadas aquí nosotros llegamos a una conclusión muy diferente. En primer lugar, no consideramos que la tonadilla desaparezca sino que se transforma, primero en las llamadas operetas y después en la zarzuela. En segundo lugar, y más importante, los elementos italianos en las operetas no van en aumento sino en franca disminución: no sólo desaparecen los recitativos sino que el final de moraleja, que era un aria da capo, se cambió por un final con moraleja, que ya no tiene esta forma ${ }^{33}$. A la par que esto ocurre se introducen innovaciones castizas como las parolas declamadas o nuevos bailes hispanos como la bolera, así como la recuperación de las seguidillas y tiranas, que en el segundo modelo tripartito (entable - coplas - final) casi habían desaparecido.

\section{PÉREZ FERNÁNDEZ (1986); LINARES Y NÚÑEZ (1998):} 189.

31 La hipótesis mediante la que señalamos que el merengue fue el resultado de binarizar las tiranas que llegaban a Venezuela a través de la tonadilla escénica requiere, sin duda, de una explicación más extensa que la que aquí, por razones de espacio, podemos dar. En la actualidad, me encuentro haciendo un estudio más detallado sobre este asunto que espero pronto vea la luz.

32 SUBIRÁ (1929): vol. 1, 206-207.

33 Es importante hacer notar, sin embargo, que sí hubo un momento en que el aumento de la influencia italiana se deja ver, como es el caso del segundo modelo tripartito, en el que el Final es un aria da capo. Sin embargo, esta influencia será rechazada en las siguientes tonadillas escénicas que en las fuentes secas eran llamadas "operetas".
Por lo tanto, si bien es cierto que la presencia de la ópera italiana produce una reacción en la tonadilla escénica, ésta no consiste en su italianización sino, muy por el contrario, en una españolización mediante la que se pretende crear una música escénica española que pueda competir con la ópera italiana. La tonadilla será así, y ahora ya coincidiendo con José Subirá, la generadora de la zarzuela del siglo XIX.

La pervivencia de la tonadilla escénica en Venezuela durante buena parte del siglo XIX hizo que se pasara de la hispanización del género a su criollización. La actualización pasará así por un proceso de apropiación que se pondrá de manifiesto en el empleo de bailes autóctonos, como el merengue. Podemos decir que este paso, fue no sólo posible, sino hasta natural, debido a que uno de los orígenes del merengue serían precisamente las tiranas que encontramos en numerosas tonadillas escénicas, las cuales, mediante un proceso de binarización, se terminaron convirtiendo en lo que se conocerá como merengue.

\section{BIBLIOGRAFÍA}

Actas y documentos que contienen las disposiciones acordadas para el examen previo de las piezas dramáticas que se representen en el teatro de esta capital desde su restablecimiento hasta el presente. Caracas, Folleto impreso, 1837.

Alonso, Celsa, La Canción Lírica Española en el siglo XIX. Madrid, ICCMU, 1998.

Andioc, René, Teatro y sociedad en el Madrid del siglo XVIII. Madrid, Editorial Castalia, 1987.

Azparren, Leonardo, El teatro en Venezuela. Ensayos históricos. Caracas, Alfadil Ediciones, 1997.

Calzavara, Alberto, Historia de la música en Venezuela. Período hispánico con referencias al teatro y la danza. Caracas, Fundación Pampero, 1987.

Capelán, Montserrat, Las reformas borbónicas y la música venezolana de finales de la colonia (1760-1821): el villancico, la tonadilla escénica y la canción patriótica. Tesis de Doctorado, Universidad de Santiago de Compostela, 2015.

Claro Valdés, Samuel, "Música teatral en América", Revista musical chilena, XXXV, 156 (Santiago de Chile, 1981): 3-20.

Fernández, Heraclio, Nuevo método para aprender a acompañar en el piano toda clase de piezas y en especial las de baile al estilo venezolano sin necesidad de ningún otro estudio y a la altura de todas las capacidades. Caracas, Imprenta de El Monitor, 1883.

García, Sonia, "Teatro y música en Cumaná. Siglo XIX", Anuario de Estudios Bolivarianos, XII/13 (Caracas, 2006), 39-63. 
Guido, Walter, "El teatro menor en Venezuela", Revista de la Sociedad Venezolana de musicología, V/8 (Caracas, 2005): 21-30.

Labrador, Germán, "Una mirada sobre la tonadilla: música, texto e intérpretes al servicio de un nuevo ideal escénico", Paisajes sonoros en el Madrid del s. XVIII. La tonadilla escénica, Madrid, Museo de San Isidro, 2003: 39-47.

Le Guin, Elisabeth, "Hacia una revalorización de la tonadilla tardía", Joaquín Álvarez Barrientos y Begoña Lolo (eds.), Teatro y música en España: los géneros breves en la segunda mitad del siglo XVIII, Madrid, UAMCSIC, 2008: 183-223.

Le Guin, Elisabeth, The Tonadilla in Performance. Lyric Comedy in Enlightenment Spain. Berkeley, Los Angeles, London, University of California Press, 2014.

Linares, María Teresa y Núñez, Faustino, La música entre Cuba y España. Madrid, Fundación Autor, 1998.

Lolo, Begoña, "Itinerarios musicales en la tonadilla escénica", Paisajes sonoros en el Madrid del s. XVIII. La tonadilla escénica, Madrid, Museo de San Isidro, 2003: 15-30.

Lolo, Begoña, "La tonadilla escénica, ese género maldito", Revista de musicología, XXV/2 (Madrid, 2002): 439469.

Lolo, Begoña, "Sainetes y tonadillas con música en el Madrid del siglo XVIII (1750-1760). Una revisión del género", Joaquín Álvarez Barrientos y Begoña Lolo (eds.), Teatro y música en España: los géneros breves en la segunda mitad del siglo XVIII, Madrid, UAMCSIC, 2008: 41-62.

Ojeda, Roberto y Capelán, Montserrat, Motetes, canciones y tonos de Atanacio Bello Montero. Caracas, Fundación Vicente Emilio Sojo, 2012.

Palacios, Mariantonia, «Rasgos distintivos del valse venezolano en el siglo XIX». Revista musical de Venezuela, 35 (Caracas, 1997): 99 -116.

Pérez Fernández, Rolando Antonio, La binarización de los ritmos ternarios africanos en América Latina. Habana, Ediciones Casa de las Américas, 1986.

Pessarrodona, Aurèlia, "La tonadilla a la Barcelona del darrer terç del Set-cents més enllà de la Casa de Comèdies", Scripta, Revista internacional de literatura $i$ cultura medieval i moderna, 3 (Valencia, 2014): 122142.
Pessarrodona, Aurélia, "Ritmos de tonadilla: algunas consideraciones a partir de la obra conservada de Jacinto Valledor", Cuadernos de música iberoamericana, 28 (Madrid, 2015): 87-114.

Romero Ferrrer, Alberto, "Los serviles y liberales o la guerra de los papeles. La constitución de Cádiz y el teatro”, Marieta Cantos, Fernando Durán y Alberto Romero (coords.), La guerra de pluma. Estudios sobre la prensa de Cádiz en el tiempo de las cortes (1810-1814), Cádiz, Universidad de Cádiz, 2009: 287-355.

Sans, Juan Francisco, Los bailes de salón en la Venezuela del siglo XIX: géneros, interpretación y estilo. Tesis de Doctorado, Universidad Central de Venezuela, 2012.

Subirá, José, La tonadilla escénica. Madrid, Tipografía de archivos, 1928, 1929, 1932.

Vera García, Rey Fernando, "Primer acercamiento al estudio de la tonadilla escénica en Nueva España durante el siglo XVIII: Edición de la letra de la tonadilla a solo: $Y a$ tienes México Grande, sobre la crítica que se hace de las cosas de teatro". Acceso: 1 de diciembre de 2016

https://www.academia.edu/30089227/La_tonadilla_escenica_en_Nueva_Espa\%C3\%B1a._Siglo_XVIII

Villabella, José Manuel: "El negrito del sainete cubano: El primer negrito (I parte)", Acceso: 14 de mayo de 2016

http://www.cubarte.cult.cu/periodico/print/articulo/7186. html

\section{FUENTES DOCUMENTALES}

Academia Nacional de la Historia (Caracas), Civiles.

Archivo General de la Nación (Caracas).

Archivo Histórico del Concejo Municipal (Caracas), Coliseo.

Biblioteca Histórica de Madrid (Madrid), Partituras manuscritas

Biblioteca Nacional de España (Madrid), Manuscritos

Biblioteca Nacional de Venezuela (Caracas), Archivo Audiovisual, Libros raros y manuscritos

Real Biblioteca de Palacio (Madrid), Partituras manuscritas

\section{PRENSA DE LA ÉPOCA}

Diario de Madrid

Gazeta de Caracas 